\title{
A Semiautomatic System for AC/DC Difference Calibration
}

\author{
KEITH J. LENTNER, MEMBER, IEEE, CLIFTON B. CHILDERS, AND SUSAN G. TREMAINE
}

\begin{abstract}
A semiautomatic ac/dc difference calibration system is described. The system operates over a frequency range of $20 \mathrm{~Hz}$ to 100 $\mathrm{kHz}$, covering the voltage range from $0.5 \mathrm{~V}$ to $1 \mathrm{kV}$. For all voltages at frequencies in the range from $20 \mathrm{~Hz}$ to $20 \mathrm{kHz}$, the total uncertainty is $50 \mathrm{ppm}$ and $100 \mathrm{ppm}$ for voltages at frequencies between $20 \mathrm{kHz}$ and $100 \mathrm{kHz}$. In addition to ac/dc difference testing, the system can be readily adapted to calibrate precision ac digital voltmeters or ac calibrators. Results of extensive intercomparison testing of the new system against a manual test system, using a multirange thermal transfer instrument as a transport standard, are reported, and the results indicate that the differences obtained are well within the combined total uncertainty limits of the two systems.
\end{abstract}

\section{INTRODUCTION}

$\mathrm{T}$ ECHNIQUES for performing precision ac/dc difference testing, not only at the National Bureau of Standards (NBS) but also in other national and industrial or governmental metrology laboratories, generally make use of manual testing methods which utilize photo-cell preamplifiers and light-beam galvanometers as voltage detectors, in conjunction with manually balanced voltage comparators [1], [2]. Careful attention to the elimination or reduction of systematic and random uncertainties in testing methods and thermoelements (TE's) has resulted in sufficient confidence in test data to allow results to be reported with total uncertainties at the 10-100 ppm level (or better, in some cases) over wide voltage and frequency ranges [3], [4]. These manual test methods, however, are very time consuming and subject to errors due to operator fatigue. To overcome these deficiencies, and to expedite test report generation, a semiautomatic ac/dc calibration system was developed.

Because of the low-level voltages that must be resolved ( 10 $\mathrm{nV}$ or less), large temperature coefficients of TE's, ac or dc source instabilities, and dc reversal differences of TE's, stringent requirements are placed upon the measurement system. In addition, light-beam galvanometers suffer the disadvantage of not being easily adaptable to automation. However, with the advent of digital electronics, low-level digital voltmeters (DVM's) are available with resolution and accuracy sufficient to meet the requirements of precision automatic ac/dc difference testing. Programmable ac and dc voltage calibrators are also available which lend themselves to adaption for automatic testing. Advances in analog/digital

Manuscript received July 24, 1980. This work was partially funded by the U.S. Department of Defense Calibration Coordination Group.

The authors are with the Electrosystems Division, National Bureau of Standards, Washington, DC 20234. interfaces have greatly improved the ease with which computers can be used to control these instruments.

The system, to be described in this paper, was designed to overcome the deficiencies of manual test methods by taking advantage of the electronics advances discussed above. Specifically, some objectives were to: 1) use commercially available programmable instruments whenever possible; 2) eliminate the need of manual data entry into a computer for data reduction; 3 ) eliminate the frequent data checks and cross checks required in manual tests; 4 ) eliminate as much operator intervention as possible during the testing procedure; 5) closely control the time interval between application of both ac and $\mathrm{dc}$ voltages to the test circuit in order to minimize drift effects; 6) provide as much computer-operator interaction as possible to simplify the testing procedure; and 7) achieve the same level of uncertainty as is presently possible with manual testing methods.

The system operates over a frequency range of $20 \mathrm{~Hz}$ to 100 $\mathrm{kHz}$, covering the voltage range from $0.5 \mathrm{~V}$ to $1 \mathrm{kV}$. For all voltages at frequencies in the range from $20 \mathrm{~Hz}$ to $20 \mathrm{kHz}$, the total uncertainty is $50 \mathrm{ppm}$ and $100 \mathrm{ppm}$ for voltages at frequencies between $20 \mathrm{kHz}$ and $100 \mathrm{kHz}$. In addition to ac/dc difference testing, the system can be readily adapted to calibrating precision ac digital voltmeters or ac calibrators.

\section{SYSTEM HARDWARE}

The hardware implementation emulates that used in manual systems at NBS. The prinicpal instrumentation difference is the use of an electronic voltage detector. Also, almost all the instruments are programmable. The major problem in hardware development was obtaining low-thermal-noise switches which could be automatically controlled. TE voltages of about $10 \mathrm{mV}$ need to be measured to within $1 \mathrm{ppm}(10 \mathrm{nV})$, and solid-state relays were found to be unsuitable since most of these devices have thermal noise of the order of $1 \mu \mathrm{V}$. The problem was solved by modifying manually operated lowthermal-noise switches. Solenoids with $24-\mathrm{V}$ operating coils were mechanically linked to the low-thermal-noise switch, and are energized by programmable relays.

Experience gained at NBS throughout many years of manual testing was used to design the remainder of the system. The switches which apply the ac or de voltages to the test circuit are relays capable of switching up to $5 \mathrm{kV}$. The $24-\mathrm{V}$ operating coils of these relays are supplied in turn by means of programmable relays. 


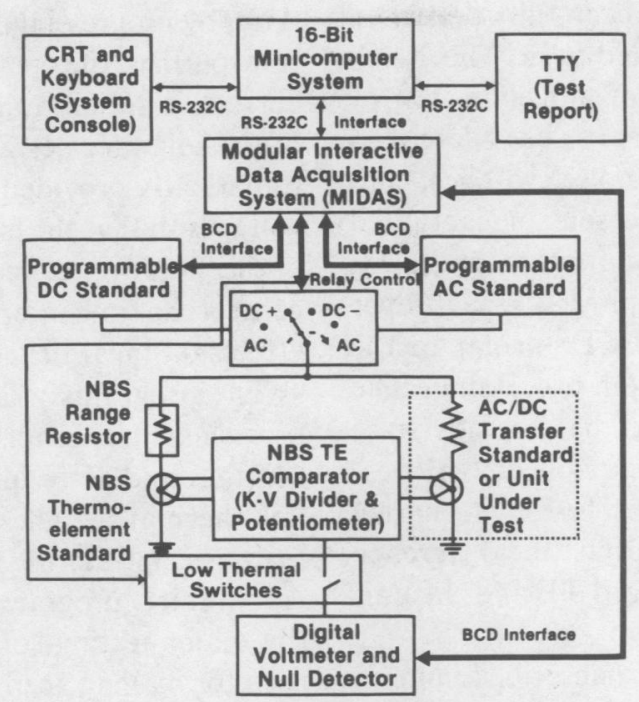

Fig. 1. Simplified system block diagram.

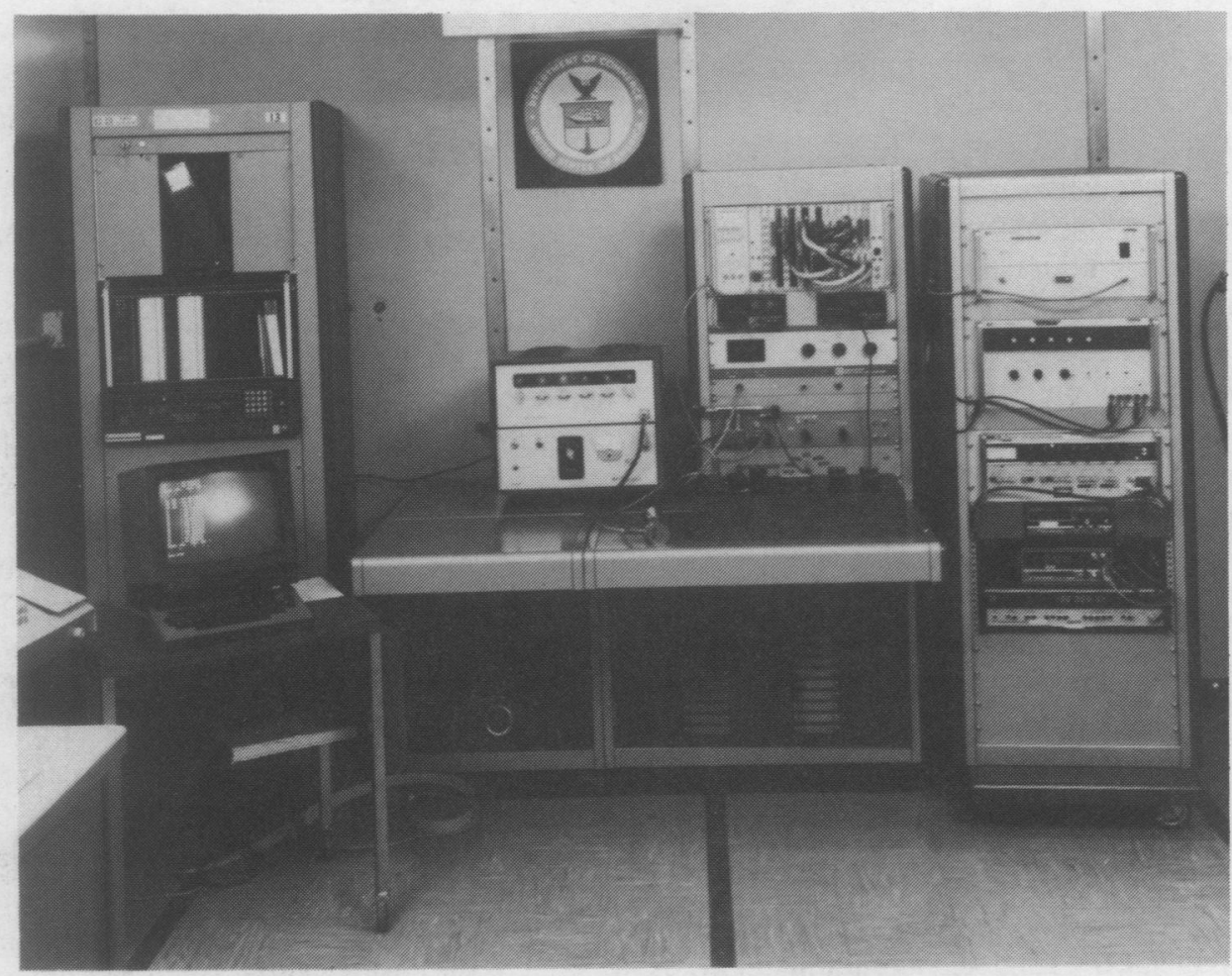

Fig. 2. Photograph of system.

A simplified block diagram of the system configuration is shown in Fig. 1, and a photograph of the system in Fig. 2. The 16-bit minicomputer serves as the system controller and has 64 kbytes of core memory with a dual floppy-disk system for program and data storage. The system console (CRT), the teletypewriter (TTY), and the Modular Interactive Data Acquisition System (MIDAS) [5] are connected to the computer by means of interfaces which conform to the EIA RS232-C [6] standard. MIDAS is a user-oriented digital interface system for data acquisition and experiment control. Its hardware is based on the CAMAC [7] standard and is composed of several functional modules which may be plugged into slots in a standard crate. A MIDAS power supply and controller are the only two additional items of equipment necessary to make MIDAS operational. Communication between the MIDAS controller and individual modules is in accordance with the ASCII [8] standard code for information interchange.

As indicated in Fig. 1, the programmable ac and dc sources and the voltage detector are controlled by binary-coded-decimal (BCD) MIDAS modules. The sequence of applying voltages to the test circuit, as indicated in Fig. 1, is ac, dc +, $\mathrm{dc}-, \mathrm{ac}$, and the ac or dc source voltage relays are controlled by means of a MIDAS relay module. The choice of this par- 
ticular voltage application sequence has been fully discussed in reference [2] and will not be reiterated here. The NBSdeveloped TE comparator (consisting of a Kelvin-Varley voltage divider and Lindeck potentiometer) has been described in the same reference. The low-thermal-noise switches, controlled by a MIDAS relay module, are solenoid actuated switches which select the proper voltage output from the TE comparator. The detector obtains readings of the proper voltages and passes these readings in BCD format to the computer where they are stored for later processing. The voltage detector is a digital voltmeter which has a steady-state input impedance of about $10 \mathrm{M} \Omega$ and is capable of $1-\mathrm{nV}$ resolution on its most sensitive $(10-\mu \mathrm{V})$ range. The Kelvin-Varley divider in the TE comparator is used to adjust the difference between the output voltages of the standard TE and the unit under test (UUT) to within $500 \mathrm{nV}$.

A typical programming sequence will be described in more detail in the next section. For simplicity in Fig. 1, two digital panel meters which monitor the voltage outputs of the dc and ac programmable standards are not shown. Also not shown is a counter used to monitor the ac standard's frequency. This equipment is visible in the system photograph (Fig. 2). The large instrument on the left of the test console table is an ac calibrator under test.

As was mentioned previously, the system can be readily adapted as well to the tasks of calibrating precision digital voltmeters or ac calibrators. In this case, the testing procedure and balance equation differ slightly from those described in reference [2]. For example, the corrections to the nominal output voltage dial settings of an ac calibrator can be determined by using the system's dc programmable standard voltage source (set to the same nominal value as the ac calibrator) and a thermal voltage converter (TVC), both having known corrections. For this case, only the Lindeck potentiometer portion of the TE comparator is used. The correction to the UUT in ppm (whether that of an external ac calibrator or the system's programmable ac standard) is given by

$$
C_{t}=\frac{V_{a}-V_{d}}{n_{s} V_{s}}+\delta_{s}+C_{\mathrm{dc}}
$$

where $\delta_{s}$ is the ac/dc difference correction of the TVC at the test frequency, $V_{a}$ is the reading of the detector (DVM) with ac voltage applied (i.e., the difference between the TE's output emf and the fixed voltage of the Lindeck potentiometer), $V_{d}$ is the average of the detector readings with direct voltage of both polarities applied, $n_{s}$ is a dimensionless factor equal to 2 if the TE has a square-law response, $V_{s}$ is the standard TE's output EMF at the applied test voltage, and $C_{\mathrm{dc}}$ is the correction to the dc voltage standard's nominal output.

\section{SYSTEM SOFTWARE}

In order to reduce the effort involved in making routine tests of TE's and TVC's, the software for the system was designed to provide as much interaction between the system console (the CRT) and the operator as possible. This interaction was used to reduce the possibility of damage to either the standard TVC or the UUT when either software or hardware failures occur.
Also, by progressing logically through the first portions of the test routines, the operator, when necessary, can take whatever corrective actions might be required. A significant advantage of the software design is that sufficient warm-up time is automatically provided between application of the various test voltages so that the effects of errors due to the warm-up drifts of the TE's are reduced. In addition, the computer can closely control the time intervals between DVM readings; hence, the effects of linear drift in the ac or dc sources are minimized.

The programs written in Basic language for this system provide the means for automatically setting the ac or dc source voltages and ac frequency, operating the voltage relays and low-thermal switches, triggering the DVM to obtain voltage readings, and storing these readings in the computer memory. About thirty programs have been written with six constituting the major testing software package. Program ACDCLV is used for making ac/dc difference tests at voltages from 0.5 to $95 \mathrm{~V}$, and ACDCHV is used for similar tests at voltages greater than $105 \mathrm{~V}$ up to $1 \mathrm{kV}$. NTSTF is used to determine a TE's dimensionless factor $n$ [1], [2] and includes provision to perform a linear least squares fit of the experimental data. ACCAL is a program used to calibrate an ac calibrator, a precision ac-digital voltmeter, or the ac source of the system in terms of a standard TVC and the system's calibrated dc supply, as discussed in the previous section.

Stability of the system's dc and ac sources can be checked by using programs called STABLV and STABHV. These programs are used at voltage ranges similar to those for ACDCLV and ACDCHV. These stability tests are useful to determine whether or not the supplies are functioning properly. Occasionally, test results appear to be erratic and the stability tests are a convenient method to ascertain whether the erratic results may be due to the ac or dc sources. Stability of the sources is measured by using a programmable $6 \frac{1}{2}$ digit DVM whose function, ranges, and reading rates are remotely controlled.

Initial stability test parameters for voltage, frequency, time between readings, and total number of readings are entered into the computer. The program then exercises complete automatic control over the ac and dc sources, the voltage relays, and the DVM. At completion, stability data including the values of minimum, maximum, range, average, standard deviation of the mean, and three times the standard deviation of the mean for both ac and dc voltage readings are printed on the TTY.

\section{OPERATION}

Since the major application of the work described in this paper has been directed toward ac/dc difference testing, a typical sequence of operations for this type of testing will be described. ACDCLV is a program which provides partial automatic control of ac/dc difference testing. The program is "partially automatic" in that an operator must manually input test parameters, switch the TE comparator to select proper TE's, and must manually balance the TE comparator's Lindeck potentiometer and Kelvin-Varley voltage divider.

Basically, ACDCLV contains three general sections. The first section provides program initialization and documenta- 
tion. The midsection includes a network of subroutines that interact with the operator. Instructions displayed on the CRT prompt the operator to: 1) key in test parameters which set ac and dc voltages and frequency; 2) switch the TE comparator to determine whether the standard TVC or UUT has the greater TE output, ensuring that the TE's are connected to the proper input terminals of the TE comparator; 3) balance one TE's output with the Lindeck potentiometer; then 4) balance the Kelvin-Varley divider. Repeatedly accessed subroutines then close and open the appropriate relays, and obtain the DVM voltage readings for four determinations of ac/dc difference at each test point. Finally, in the third section, statistical calculations are performed, and the results are printed on the TTY. Options are provided, and the operator can choose whether to stop, repeat a test, or change parameters (either voltage or frequency) and run another test. This program is used for voltages $\leq 95 \mathrm{~V}$.

Since the MIDAS interface does not have isolated analog and digital grounds, it was necessary to develop a separate software package for high-voltage testing. At high voltages, it was found that the opening or closing of either the ac or dc voltage relays introduced transient voltage surges into the MIDAS digital circuitry. The MIDAS controller interpreted these surges as voltage level changes identical to programmed input commands. The result was that incorrect relays might be opened or closed, the voltage detector might be falsely triggered to obtain a reading, or any other of a series of unwanted events might occur. Hence, the ACDCHV program was developed to ensure that no high-voltage supplies are turned on whenever a voltage relay is opened or closed. The specific procedures include:

1) Input test number, voltage, frequency, observer's name, and code for the standard TVC used.

2) Visually check to see that both software and hardware are operating correctly by checking the system's digital panel meters and frequency meter for correct readings of the outputs from the ac and dc sources. If the outputs are incorrect, try again.

3) Manually set the TE comparator to its high TE voltage position and switch the voltage detector to its $10-\mathrm{mV}$ range. The program automatically closes the dc voltage relay and a low-thermal-noise switch, turns on the dc supply, and triggers the detector to obtain the TE voltage output reading.

4) Switch the TE comparator to its low TE voltage input position.

The program then commands the detector to read the voltage. The values of the high- (from the previous step) and lowvoltage readings are compared. If the values are incorrect, the operator is instructed to interchange the two TVC inputs after the dc voltage has been turned off. The program automatically stores the value of $E_{\text {low }}$, since that value is used in the balance equation [2, appendix].

5) Switch the TE comparator to the high TE input position, turn on the Lindeck potentiometer, change the detector to its $10-\mu \mathrm{V}$ range, and manually balance the potentiometer.

6) The first low-thermal-noise switch is opened and a second closed. The operator now manually balances the KelvinVarley divider for a near null reading on the detector. The dc source is automatically turned off, and the dc voltage relay opened.

7) The ac voltage relay is closed, the ac supply turned on, and its voltage is automatically incremented or decremented until the UUT's TE output voltage is $\leq 1 \mu \mathrm{V}$ of its value with dc voltage applied as in step 6.

8) At this point, the manual initialization process is complete, and the test proceeds automatically under program control. A sequence of near null voltage readings is obtained by the null detector when ac, dc,$+ \mathrm{dc}-$, and ac voltages are applied to the standard TVC and UUT. These four voltage readings are each obtained from the average of ten readings of the DVM. The standard deviation of the ten readings is computed and, if this value is $<200 \mathrm{nV}$, the program continues. If the standard deviation is $\geq 200 \mathrm{nV}$, due to drifts in either the standard TVC or the UUT, or the ac or dc sources, or any of these effects combined, the series of ten readings is repeated. After five attempts, if the DVM readings have not stabilized, the test is aborted.

9) The sequence of readings with ac, dct, dc-, and ac voltages applied constitutes one determination of the ac/dc correction to the UUT. The process is continued for four determinations; the average value of four determinations is computed and printed on the TTY. The operator then may elect to repeat the test, change voltage range or frequency, or stop the test.

The system was intended to calibrate high-quality TVC's, and it was not necessary to add additional balancing steps to eliminate uncertainties due to large dc reversal difference (i.e., difference between the dc direct and reverse voltages required for the same TE EMF output of the UUT). For the worst case, using 2.0 and 1.6 as the two extremes of the characteristic $n$ which may occur for the standard and UUT TE's, it can be shown [4] that systematic uncertainties of less than one-fourth the UUT's dc reversal difference will occur. Minor software changes can eliminate this systematic source if large reversal differences are anticipated. The changes would amount to adjusting the ac voltage (cf. step 7 above) for a TE output equal to that when dc reversed voltage is applied to the test circuit. The only penalty for such a change in the operating procedure would be an increase in testing time of perhaps one or $2 \mathrm{~min}$ (for four determinations of ac/dc difference).

There is also the possibility of a systematic uncertainty if the ac or dc voltage sources drift during a sequence of four determinations. However, for the same extremes of $n$, the uncertainty is only $10 \mathrm{ppm}$ if either source drifts by as much as $100 \mathrm{ppm}$. The STABLV and STABHV programs readily provide information about the stabilities of the sources.

\section{MEASUREMENT RESULTS AND UNCERTAINTIES}

There are many error sources which determine the uncertainty of ac/dc difference measurements of the type described in this paper. Similar manual systems have been in operation for many years at NBS and their uncertainties have been documented [1]-[3]. DC reversal difference of a TE, selfheating and ambient temperature effects, drift effects due to nonequal time intervals during the voltage measurements, unbalanced lead impedances, and induced voltages from 
TABLE I

RANGE OF AC/DC DIFFERENCES FOR TRANSPORT STANDARD (ppm)

\begin{tabular}{|c|c|c|c|c|}
\hline \multirow{3}{*}{$\begin{array}{l}\text { Vol tage } \\
\text { Range }\end{array}$} & \multirow{3}{*}{$\begin{array}{l}\text { Test } \\
\text { Voltage } \\
\text { Volts } \\
\end{array}$} & \multicolumn{3}{|c|}{ Test Frequency $(\mathrm{kHz})$} \\
\hline & & 20 & 50 & $\underline{100}$ \\
\hline & & & & \\
\hline $0.25-0.5$ & 0.5 & 18 & 19 & 23 \\
\hline $0.5-1$ & 1 & 9 & 2 & 4 \\
\hline $1-2$ & 2 & 1 & 6 & 9 \\
\hline $2-4$ & 3 & 2 & 0 & 0 \\
\hline $4-8$ & 8 & 0 & 1 & 3 \\
\hline $8-16$ & 16 & 8 & 15 & 3 \\
\hline $16-32$ & 30 & 8 & 10 & 9 \\
\hline $32-64$ & 50 & 3 & 3 & 10 \\
\hline $64-125$ & 98 & 2 & 6 & 12 \\
\hline $125-250$ & 200 & 4 & 2 & 14 \\
\hline $250-500$ & 500 & 4 & 50 & 39 \\
\hline $500-1000$ & 500 & 22 & 54 & 147 \\
\hline $500-1000$ & 1000 & 17 & 70 & -- \\
\hline
\end{tabular}

electromagnetic fields are some of the more common sources of error. The process of determining an estimated total uncertainty for a system is based upon experience with the system, careful measurements of standards with accurately known corrections, or intercomparison tests of two independent test methods [3]. The latter procedure, involving comparison of a well-characterized manual system with the new one, was followed, since the uncertainty sources are believed to be the same in both systems.

The system performance was verified by an extensive testing procedure which involved the use of a multirange thermal voltage converter instrument as a transport standard. The transport standard was first tested for ac/dc difference corrections at various combinations of applied voltages and frequencies using the MIDAS-based system. It was then tested, at similar voltages and frequencies with the same standard TVC, using the manual testing techniques which have been used for many years at NBS. A third test was performed, again using the MIDAS system. Finally, the MIDAS system was disassembled, physically relocated, reassembled, and tested for proper operation. Then the transport standard was tested a fourth time, again using the MIDAS system. It should be noted that not all voltage range-frequency combinations were tested for all four tests. However, in those cases where fewer than four tests were performed, the results indicated sufficiently small differences between at least two independent test methods to establish that further testing was not warranted. The results of these independent intercomparison tests are summarized in Table I. In evaluating these results, it must be remembered that, for calibrating multirange instruments like the transport standard, the manual test system has uncertainties of the same magnitude as the semiautomatic system. Hence, combined total uncertainties of the order of 100 and $200 \mathrm{ppm}$ are possible depending upon the test frequency.

In Table I, the column listing "Voltage Range" refers to the input voltage range of the transport standard, the "Test
Voltage" is the actual test voltage applied, and the different columns for frequency are the actual test frequencies used. The numbers listed under the frequency columns are the maximum differences (in ppm) of the calibrated ac/dc difference corrections for the transport standard as determined for at least two, and up to a maximum of four, of the test conditions described above. That is, the numbers reported are the range (magnitude of the maximum minus the minimum) of the ac/dc difference corrections calculated for each test voltage and frequency. The total elapsed time between the first and final tests of the transport standard was about eight weeks.

From the test results at $20 \mathrm{kHz}$, the average value of the range of ac/dc differences for all voltages is only $8 \mathrm{ppm}$. At 50 $\mathrm{kHz}$ the average value of the range is $19 \mathrm{ppm}$, and at $100 \mathrm{kHz}$, where skin effects and stray capacitance effects predominate, the largest difference (at $500 \mathrm{~V}$ ) was $147 \mathrm{ppm}$. However, this large range is within the expected combined total uncertainty of $200 \mathrm{ppm}$ for the two systems. Even with this one range greater than $100 \mathrm{ppm}$, the average value of the range at 100 $\mathrm{kHz}$ is still only $23 \mathrm{ppm}$. No intercomparison results were obtained at $1000 \mathrm{~V}, 100 \mathrm{kHz}$ since the high-voltage amplifiers in both systems could not supply sufficient power at that voltage-frequency combination.

\section{CONCLUSIONS}

The results of the work reported in this paper indicate that it is possible to measure ac/dc differences semiautomatically with uncertainties about the same as those obtained with manual testing techniques. The major advantage of the system appears to be the elimination of most of the tedium involved in manual testing, in which deflections of light beam galvanometers must be observed and recorded.

For future work, it appears that improvements in system operation can be obtained by development of self-balancing TE comparators. Beyond that, it may be possible, by taking advantage of improvements in voltage detector resolution, desktop computers, and system components whose operation is compatible with new digital/analog interfaces, to make readily available the hardware and methodology for improved ac/dc difference testing techniques.

\section{ACKNOWLEDGMENT}

The authors are indebted to E. S. Williams for his many valuable contributions to the work reported in this paper. He designed and built the voltage and low-thermal-noise switch modules and provided much of the initial software which eventually became part of the total programming package required for system operation. R. E. Kleimann, S. C. Bailey, and W. C. Caterisano each contributed portions to some of the computer programs.

\section{REFERENCES}

[1] F. L. Hermach and E. S. Williams, "Thermal converters for audio-frequency voltage measurement of high accuracy," IEEE Trans. Instrum. Meas., vol. IM-15, pp. 260-268, Dec. 1966.

[2] E. S. Williams, "Thermal voltage converters and comparator for very accurate ac voltage measurements," Nat. Bur. Stand. J. Res., vol. 75C, 
pp. 145-154, Dec. 1971

[3] F. L. Hermach, "AC-DC comparators for audio-frequency current and voltage measurements of high accuracy," IEEE Trans. Instrum. Meas., vol. IM-25, pp. 489-494, Dec. 1976.

[4] R. S. Turgel, "A comparator for thermal ac-de transfer standards," ISA Trans., vol. 6, no. 4, pp. 286-292, 1967.

[5] C. H. Popenoe, M. S. Campell, "MIDAS Modular Interactive Data Acquisition System-description and specifications," Nat. Bur. Stand. Tech. Note 790, U.S. Gov. Printing Office, Washington, DC 20402 (Aug. 1973).
[6] "Interface between data terminal equipment and data communication equipment employing serial binary data interchange," EIA RS-232-C Electron. Ind. Ass., 2001 Eye St., N.W., Washington, DC 20006 (Aug. 1969).

[7] "IEEE standard modular instrumentation and digital interface system (CAMAC)-computer automated measurement and control," IEEE Stand. 583-1975, IEEE, 345 E. 47 St., New York, NY 10017.

[8] "American National Standard Code for Information Interchange," ANSI X3.4-1977, Amer. Nat. Stand. Inst., Inc., 1430 Broadway, New York, NY 10018.

\title{
A Thermoelement Comparator for Automatic AC-DC Difference Measurements
}

\author{
EARL S. WILLIAMS
}

\begin{abstract}
AC-DC differences in thermal voltage converters (TVC's) are determined relative to a similar instrument, from imbalance EMF's measured in a divider circuit to which both instrument outputs are connected. These EMF's are more stable than the input voltages to the converters, because input voltage fluctuations produce nearly equal proportional changes in the converter outputs. The divider balance is therefore affected only slightly. The divider and a Lindeck potentiometer for monitoring the test instrument output are driven by stepping motors and balanced automatically. AC and DC voltages are applied by programmable power supplies whose outputs are adjusted, in response to imbalance EMF's in the potentiometer circuit, to produce equal output from the test instrument. The system automatically calibrates one voltage range at one or more frequencies, but requires an operator to change ranges and to enter the test parameter at a computer terminal.
\end{abstract}

\section{INTRODUCTION}

$\mathrm{A}^{\mathrm{c}}$ C VOLTAGE IS measured most accurately at the present time when it is compared directly with a dc reference voltage using a thermal voltage converter (TVC). These instruments, which are also called thermal transfer voltmeters, consist essentially of ranging resistors and a thermoelement (TE), a short straight electrical heater whose temperature is sensed by a thermocouple or a thermopile. Multirange TVC's usually include a balancing EMF source and a null detector for monitoring the TE output.

The reference voltage $V_{d}$ is usually an accurately measured "round number" value which is nominally equal to the ac voltage $V_{a}$ to be measured. The proportional difference between the two voltages $\left(V_{a}-V_{d}\right) / V_{d}$ is measured by observing

Manuscript received June 28, 1980.

E.S. Williams is with the National Bureau of Standards, Washington, DC 20234. the output $E$ of the TVC, and changes in output as the TVC is connected alternately to the two voltages. Both polarities of dc voltage are generally used because the TE in most TVC's has a significant reverse dc difference. When the difference between the ac and dc voltages is small

$$
\frac{\left(V_{a}-V_{d}\right)}{V_{d}}=\frac{\left(E_{a}-E_{d}\right)}{n E} .
$$

$E_{a}$ is the EMF observed when ac voltage is applied to the TVC, $E_{d}$ is the average of EMF's observed when the forward and reverse dc voltage is applied, and the factor $n$ relates small changes in TE heater current to the corresponding changes in output EMF.

Ideally the TE has a square-law response with $E=k I^{n}(n$ $=2$ and $k$ constant for small changes in $I$ ). However, the value of $n$ deviates from 2 as the heater current is increased and is usually in the range of 1.7 to 1.9 at the rated current of the TE. Values of $n$ must be determined at several levels by applying known voltage changes $\Delta V / V$ to the TVC and observing the output $E$ and changes in output $\Delta E$. The equation is very much like (1) above:

$$
\Delta V / V=\Delta E / n E
$$

or

$$
n=\frac{\Delta E / E}{\Delta V / V}
$$

AC voltage measurements, as described above, usually require the application of a correction $(\delta)$ for the ac-dc difference of the TVC. Such corrections are needed due to inductance in the ranging resistors and range selecting switches and 